\title{
Prevention of cartilage dehydration in imaging studies with a customized humidity chamber
}

\section{Other Conference Item}

Author(s):

Choo, Ryan J.; Steiner, Thomas H.; Firminger, Colin R.; Müller, Ralph (D); Stok, Kathryn S.

Publication date:

2012

Permanent link:

https://doi.org/10.3929/ethz-a-007365455

Rights / license:

In Copyright - Non-Commercial Use Permitted 


\title{
PREVENTION OF CARTILAGE DEHYDRATION IN IMAGING STUDIES WITH A CUSTOMIZED HUMIDITY CHAMBER
}

\author{
Ryan J. Choo, Thomas H. Steiner, Colin R. Firminger, Ralph Müller, Kathryn S. Stok
}

Institute for Biomechanics, ETH Zurich, Zurich, Switzerland

\section{Introduction}

Quantitative three-dimensional imaging methods allow rapid and comprehensive evaluation of cartilage and bone in animal models, used for drug development and related research in arthritis.

When imaging fresh cartilage tissue using synchrotron radiation microcomputed tomography $(\mathrm{SR}-\mu \mathrm{CT})$, one problem is tissue dehydration causing movement artefact in the resulting images. This is especially noticeable at high resolutions (6 $\mu \mathrm{m}$ and higher), and occurs even in fast $(<5$ minute) scans. A humidity chamber was designed to counteract this problem and maintain cartilage hydration during scanning.

The goal of this work was to evaluate the performance of the humidity chamber. Quantitative measures of cartilage volume were made to test dehydration over scan time, and at various umidity (RH\%) levels.

\section{Materials and Methods}

The humidity chamber holder houses a temperature/humidity sensor and heater that are used to monitor and regulate test conditions. Specimens are placed in a radio-translucent measurement tube which is screwed into the holder. This creates a seal which prevents moisture inside the chamber from escaping. The measurement tube is coated with lead film to protect the temperature/humidity sensor from radiation.

Three mouse knees were placed in the humidity chamber and scanned with SR- $\mu \mathrm{CT}$ at the Swiss Light Source, using an isotropic voxel size of 3.7 $\mu \mathrm{m}$. The samples were immersed in $20 \%$ Hexabrix $^{\mathrm{TM}}$ (Mallinckrodt, USA), a staining agent to help visualise cartilage.

This study was divided into two experiments. Firstly, two knees were scanned using a low RH\% for 20 minutes in 10 minute intervals to test the change in volume with time due to dehydration. Secondly, a knee was scanned at decreasing $\mathrm{RH} \%$ of $97 \%, 92 \%$ and $87 \%$, to test for a humidity level where volume drops away.

Bone and cartilage from the resulting images were manually segmented from the epiphyseal region of the tibia. Morphometric analysis was performed to determine the cartilage volume in each scan. Cartilage volume was then plotted against (1) time, and (2) $\mathrm{RH} \%$.

\section{Results}

Evidence of tissue dehydration is shown in fig. 1A; immediate loss of cartilage volume is observed when scanned at a low $\mathrm{RH} \%(<90 \%)$ over a long period. In this scenario, dehydration occurs at approx $0.2 \mu \mathrm{m} / \mathrm{min}$. A loss of volume can also be attributed to scanning at decreasing $\mathrm{RH} \%$ (fig. 1B).

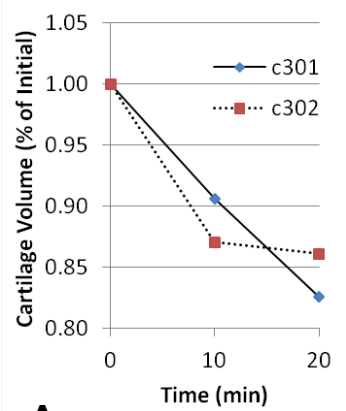

A.

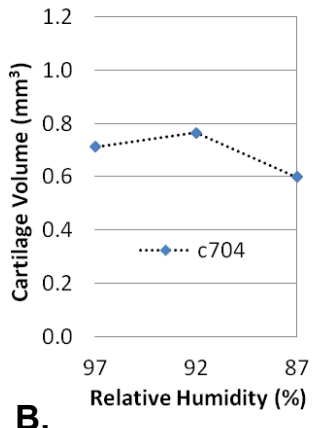

B. mouse knees. (B) Cartilage volume against relative humidity for a mouse knee.

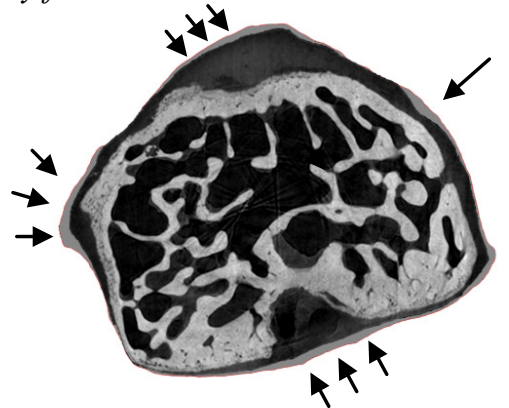

Figure 2: Overlay of the images at $87 \%$ and $92 \%$ relative humidity for a sample. The difference in cartilage thickness is shown by the grey border (indicated by arrows).

\section{Discussion}

The results clearly show that loss of cartilage volume is associated with scanning at low humidity (fig. 2). This effect can be avoided by using the humidity chamber for SR- $\mu \mathrm{CT}$ imaging above $92 \%$, since maintaining tissue hydration will reduce or eliminate cartilage shrinkage. Confirmation of these results is planned in ongoing work; since this study is limited due to restricted access to facilities for high-resolution SR- $\mu$ CT.

\section{Acknowledgement}

Swiss Commission for Technology and Innovation (CTI), Grant No 9853.1. 